\title{
SOSTENIBILIDAD Y CADENAS AGROPRODUCTIVAS DE CACAO EN EL PERÚ PERSPECTIVAS DESDE LAS REGIONES PIURA Y SAN MARTÍN
}

\author{
Lourdes Amparo Lares Acero ${ }^{1}$
}

\begin{abstract}
RESUMEN
La sostenibilidad de la cadena de valor de cacao en el Perú resulta importante, porque se encuentra relacionada directamente con el crecimiento económico de las familias productoras de cacao y pueden tener la posibilidad de salir de la pobreza. Los factores identificados en las cooperativas APPROCAP en Piura y ACOPAGRO en San Martín, que favorecen la sostenibilidad de esta cadena, son la diversidad de material nativo y su alta calidad, respectivamente. Ambas características son reconocidas en mercados especializados que pagan mejores precios por estos cacaos, lo que ha favorecido además su producción continua. El desarrollo de capacidades a los socios productores, así como la asociatividad son también factores importantes en ambas cadenas que han favorecido su sostenibilidad. Las políticas nacionales sobre cacao en el Perú deben poner más atención a sus diferentes cacaos a pesar de que muchos de ellos, los nativos, tengan menos volumen productivo pero mucho valor de biodiversidad y a la vez económico.
\end{abstract}

Palabras clave: Sostenibilidad. Cadenas agroproductivas. Cooperativas. Cacao.

\section{RESUMO}

A sustentabilidade da cadeia de valor do cacau no Peru é importante, porque está diretamente relacionada ao crescimento econômico das famílias produtoras de cacau e elas podem ter a possibilidade de escapar da pobreza. Os fatores identificados nas cooperativas APPROCAP em Piura e ACOPAGRO em San Martín, que favorecem a sustentabilidade dessa cadeia, são a diversidade de materiais nativos e sua alta qualidade, respectivamente. Ambas as características são reconhecidas em mercados especializados que pagam melhores preços por esses cacaus, o que também favoreceu sua produção contínua. O desenvolvimento da capacidade dos parceiros produtores, bem como a associatividade, também são fatores importantes nas duas cadeias que favoreceram sua sustentabilidade. As políticas nacionais de cacau no Peru devem prestar mais atenção a seus diferentes cacaus, apesar de muitos deles, os nativos, terem menos volume produtivo, mas muita biodiversidade $\mathrm{e}$ valor econômico.

Palavras-chave: Sustentabilidade. Cadeias agro-produtivas. Cooperativas. Cacau

Submetido em: 14.01 .2020

Aprovado em:16.03.2020

\footnotetext{
${ }^{1}$ Universidad Nacional Mayor de San Marcos. Investigadora en cadenas agro-productivas, circuitos cortos de comercialización y desarrollo sostenible. Licenciada en Antropología (PUCP) y egresada de la Maestría en Estudios Amazónicos (UNMSM). E-mail: lares.lourdes@gmail.com
} 


\section{INTRODUCCIÓN}

El Perú posee el $60 \%$ de los grupos genéticos de cacao del mundo. Estos se encuentran ubicados principalmente en la Amazonía alta y en la costa norte de nuestro país.

La sostenibilidad de esta cadena agroproductiva se va construyendo durante los diferentes procesos y etapas de su desarrollo, la misma que se relaciona directamente también al crecimiento económico de las familias productoras y sus organizaciones.

La importancia de este producto en nuestro país radica además en que mueve la economía de más de 90 mil familias de agricultores en 144,276 hectáreas (LARES et al., 2019) con una producción de 134,676 toneladas en el año $2018^{2}$. Además, según la International Cocoa Organization (ICCO), Perú es el tercer productor y exportador de cacao en Latinoamérica, después de Brasil y Ecuador y el segundo productor mundial de cacao orgánico (BANCO MUNDIAL, 2017).

Así, conocer las diferentes perspectivas de sostenibilidad de una misma cadena agroproductiva en diferentes regiones y contextos, permitirá tener un conocimiento inicial de sus diferentes necesidades y cómo deberían abordarse.

Este artículo busca mostrar brevemente las similitudes y diferencias en las perspectivas de sostenibilidad de las cadenas agroproductivas de cacao de dos organizaciones de productores en las regiones de Piura y San Martín: la cooperativa Asociación de Pequeños Productores de Cacao de Piura - APPROCAP y la Cooperativa Agraria Cacaotera ACOPAGRO.

Ambas organizaciones han pasado por diferentes procesos de formación y de participación en el mercado, con diferentes actores vinculados que forman parte de sus respectivas cadenas agro-productivas.

Para ello, se indicará cuál es el enfoque actual de las políticas estatales sobre cacao en nuestro país. Posteriormente, se mostrarán las diferentes miradas que abordan los conceptos de sostenibilidad y cadenas agroproductivas, que servirán como base para analizar las diferencias y similitudes en ambas cooperativas. Las dos secciones finales del artículo mostrarán las conclusiones y referencias del mismo, respectivamente.

\footnotetext{
2 https://gestion.pe/economia/minagri-plan-reducir-contenido-cadmio-cultivos-cacao-267617?fbclid=IwAR0kKS6WHBL2S1Tlv6pYRK63WTvEMOOock-c2XU7-FvV3bJzDvGU-NrpJc
} 


\section{POLÍTICAS ESTATALES SOBRE CACAO EN EL PERÚ}

El monitoreo agroclimático del cultivo de cacao que hace el MINAGRI está enfocado principalmente en las regiones amazónicas como San Martín, Huánuco y Ucayali ${ }^{3}$. De igual manera, las Mesa Técnica de cacao a nivel nacional, así como el gremio de productores de cacao, Asociación Peruana de Productores de Cacao - APPCACAO cuentan mayoritariamente con socios de regiones amazónicas entre sus miembros, quienes son los principales interlocutores ante el Estado y la cooperación internacional.

Esto se debe a que el $93 \%$ de la producción nacional de cacao se concentra en 7 de las 16 regiones donde se cultiva cacao ${ }^{4}$ y donde la región San Martín ocupa el primer lugar con el $42 \%$ y Piura el 2\% de la producción a nivel nacional (LARES et al., 2019). Cabe resaltar además que un tercio de la producción nacional cuenta con certificación orgánica y de Comercio Justo.

Así, la mirada desde el Estado en torno a la actividad productiva del cacao resalta su enfoque amazónico ya que este cultivo está "ubicado principalmente en Ceja de Selva, constituyendo una actividad económica inclusiva al desarrollarse principalmente en zonas ex cocaleras, comunidades nativas y zonas de frontera"5 (MINAGRI, 2018).

Asimismo, luego de la consolidación de esta cadena en las regiones de la Amazonía Norte y Centro, a partir de los programas de desarrollo alternativo desde los años noventa, el Gobierno Central ha empezado a replicar estos resultados en los Valles de los Ríos Apurímac, Ene y Mantaro (VRAEM), en las regiones Ayacucho y Junín. Esto con el fin de eliminar el narcotráfico a partir de la comercialización de productos locales como cacao y café.

Los resultados de los programas de desarrollo alternativo en el Perú con cacao indican que el $64 \%$ de las hectáreas de producción actual de este producto proviene de los programas liderados por la Comisión Nacional para el Desarrollo y Vida sin Drogas (DEVIDA) y la cooperación norteamericana a través de USAID (WILDLEY, 2016).

\section{SOSTENIBILIDAD Y CADENAS AGROPRODUCTIVAS EN PIURA Y SAN MARTÍN}

\subsection{SOSTENIBILIDAD}

\footnotetext{
${ }^{3}$ https://www.minagri.gob.pe/portal/monitoreo-agroclimatico/cacao-2018?start=20

${ }^{4}$ https://agraria.pe/noticias/el-93-de-la-produccion-peruana-de-cacao-se-concentra-en-7-re-16171

Junín (19\%), Cusco (9\%), Ucayali (8\%), Huánuco (6\%), Ayacucho (5\%) y Amazonas (4\%).

${ }^{5}$ https://cdn.www.gob.pe/uploads/document/file/229452/rm451-2018-minagri.pdf
} 
La idea de sostenibilidad aparece por primera vez en 1713 en Alemania, según el historiador Ulrich Grober (2012). Una de las primeras referencias a este concepto apareció en un documento forestal ${ }^{6}$, en el contexto de la creciente demanda de madera para alimentar las plantas de fundición para martillos en la región de Sajonia. Aquí, Hans Carl von Carlowitz criticó la visión de corto plazo que permitía que los bosques se talaran con este fin. En ese sentido, planteó la interrogante de cómo se podría organizar el cultivo de la madera para hacer posible su uso continuo, constante y sostenible, debido a la importancia que tenía para su país (BRIGHTMAN et al., 2017).

En el siglo XXI, las Naciones Unidas definen el concepto de sostenibilidad haciendo referencia a la "satisfacción de necesidades del presente sin comprometer la capacidad de las generaciones futuras para satisfacer sus propias necesidades". Con esto se asume que los recursos son limitados y deben usarse considerando las consecuencias de su uso. Posteriormente, su uso también se asocia al concepto de desarrollo y de "capitalismo ecológico", para reducir los efectos negativos sobre el medio ambiente causados por actividades productivas, pero sin alterar la lógica de acumulación.

De esta manera, vemos que el término "sostenibilidad" ha pasado por tener características de interés social, productivo y de conservación a estar dominado por una preocupación con consideraciones económicas justificada en un interés por el cuidado del medio ambiente. En ese sentido, el discurso oficial muestra una tendencia por querer alcanzar un equilibrio con las dimensiones sociales y ecológicas, de manera compatible con el crecimiento económico.

La antropología de la sostenibilidad, por su parte, agrega que se deben estudiar procesos culturales desde diferentes perspectivas basada en los diferentes intereses y necesidades, en lugar de los intereses universales de una tradición dominante. En este mismo sentido, Del Pozo (2016) señala que la sostenibilidad se basa en alcanzar una nueva conciencia colectiva de los efectos de nuestras acciones a largo plazo. De esta manera, la sostenibilidad debe ser entendida como el proceso para facilitar condiciones de cambio construyendo y apoyando la diversidad (BRIGHTMAN et al., 2017).

De esta manera, para este artículo utilizaremos el término sostenibilidad haciendo referencia a cómo estas se pueden reproducir por sus propias características, de manera continua y a la vez vinculado al crecimiento económico.

\footnotetext{
6 "Sylvicultura oeconomica” (1713)
} 


\subsection{CADENAS AGROPRODUCTIVAS}

El concepto de cadenas agroproductivas en el Perú se relaciona a un enfoque de oportunidad de mercado. En ese sentido, el Ministerio de Agricultura y Riego (MINAGRI, 2007) lo define como "el sistema que agrupa a los agentes económicos interrelacionados por el mercado y que participan articuladamente en un mismo proceso, desde la provisión de insumos hasta el consumo final [...] en forma sostenida y rentable, basados en principios de confianza y equidad". Por otro lado, Paredes y Fort (2018) en su balance sobre cadenas de valor y el desarrollo rural en el Perú la definen como "la cadena destinada a mercados diferenciados y cómo estos atributos condicionan las oportunidades de vinculación con pequeños productores, así como los efectos económicos que resultan de su participación”.

Así, los factores para el surgimiento de estas cadenas en el Perú fueron la identificación del potencial productivo y comercial, y la articulación de pequeños productores impulsadas por actores privados como empresas, ONG y la cooperación internacional (PAREDES et al., 2018). En muchos casos, esta articulación se produce también con organizaciones de productores como cooperativas o asociaciones.

Asimismo, los productos de una cadena agroproductiva diferenciados en mercados nicho, y que obtienen mejores precios por ello, fortalecen las cadenas agroproductivas en mercados especializados como en el Biocomercio ${ }^{7}$; esto por su gran potencial para la sostenibilidad ambiental, la generación de empleo y la reducción de la pobreza (LARES et al., 2019).

Así, nuestro análisis sobre la sostenibilidad de las cadenas agroproductivas de cacao en estas cooperativas se centrará en su capacidad para auto-mantenerse en términos financieros, haciendo énfasis en lo que es beneficioso económicamente en el tiempo para los agricultores. Esto porque la sostenibilidad de las cadenas agroproductivas de cacao se traduce en crecimiento económico para las familias productoras de cacao y sus organizaciones (LARES et al., 2019). También porque hace referencia a la vinculación de actividades productivas y de valor agregado que además genere un beneficio ambiental, cultural y social.

Esto resulta relevante porque a pesar de que el crecimiento de una cadena de valor sea una señal de que puede sostenerse económicamente, el término sostenibilidad se refiere también a la vinculación de las actividades productivas y de agregación de valor con su

\footnotetext{
${ }^{7}$ Según la legislación peruana, es la actividad que a través del uso sostenible de los recursos nativos de la biodiversidad promueve la inversión y el comercio, apoyando el desarrollo de la actividad económica y generando valor agregado de productos para el mercado local e internacional con criterios de equidad social y rentabilidad económica (Reglamento de Ley sobre conservación y aprovechamiento sostenible de la diversidad biológica D.S. 068-2001-PCM).
} 
contexto ambiental y social, de manera a crear beneficios también en estos ámbitos (VIDAL, 2015), como en el caso de la conservación de los cacaos nativos en Piura y los sistemas agroforestales en San Martín.

Finalmente, nuestro análisis tendrá también en cuenta cómo las cadenas agroproductivas analizan su sostenibilidad a partir de la atención al desarrollo de capacidades. Para ellas, la experiencia de asociatividad y de vinculación con la cooperación internacional, ha sido un elemento clave para la articulación a los mercados nicho (MINAGRI, 2015) para, mediante la acción colectiva, conseguir mayores ingresos económicos (FORT et al., 2015).

\section{EL CASO DE LAS COOPERATIVAS CACAOTERAS APPROCAP Y ACOPAGRO}

\subsection{Cooperativa APPROCAP - Piura}

La cooperativa Asociación de Pequeños Productores de Cacao de Piura - APPROCAP fue fundada en el año 2003 y se encuentra ubicada en el distrito de San Juan de Bigote, provincia de Morropón. Actualmente cuenta con 242 socios y 250 hectáreas de producción de cacao. El promedio por año de su producción es menos de $500 \mathrm{~kg}$ por hectárea. La región Piura tiene 1,429 hectáreas de cacao nativo que producen 599 toneladas al año en total según el INEI (2018).

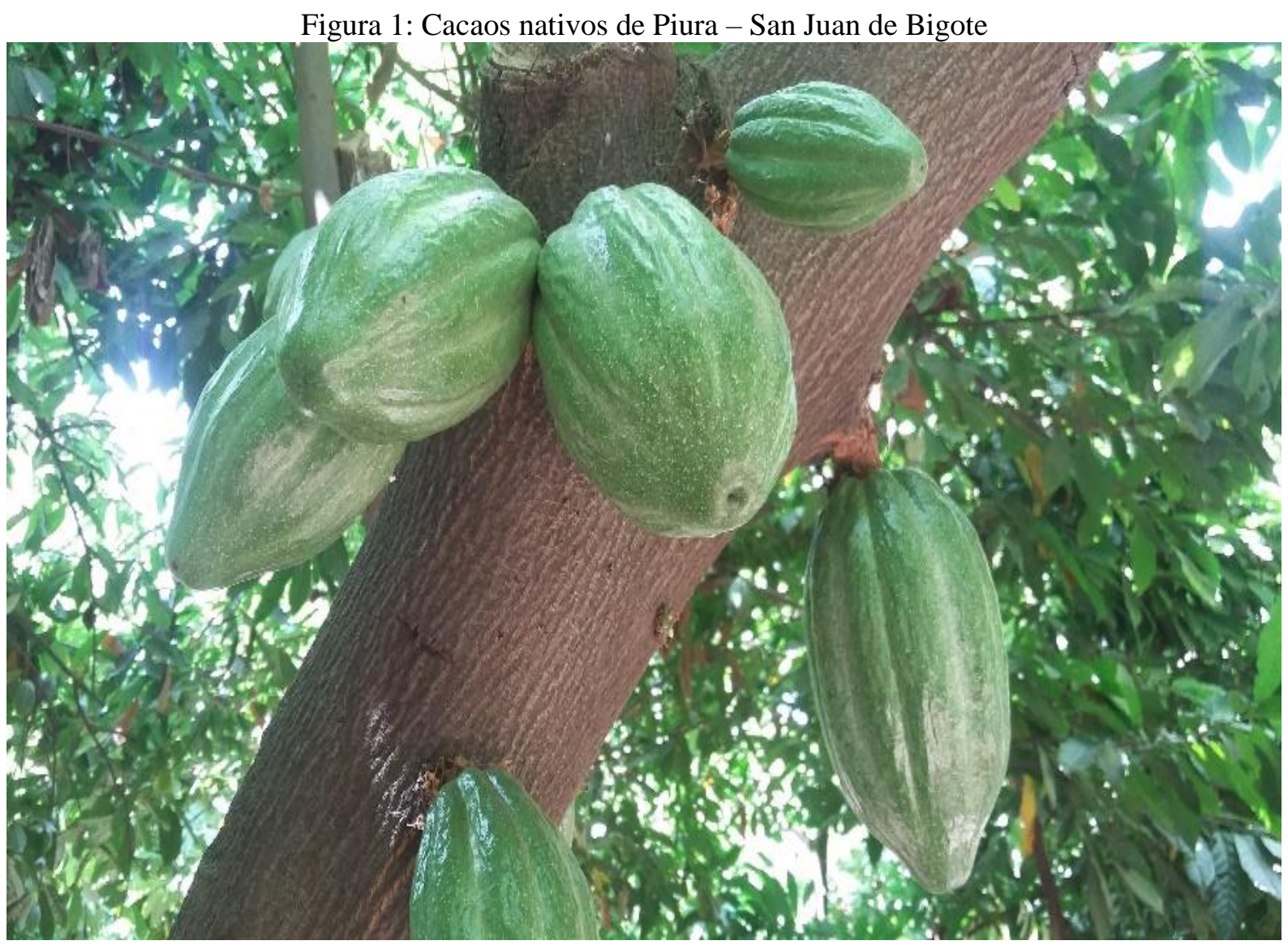


Comparado con otras regiones, los niveles de producción de cacao en Piura no son tan altos, pero sí lo son para el mercado orgánico ${ }^{8}$, por lo que actualmente es una opción importante para el desarrollo económico de las familias productoras.

Asimismo, investigaciones previas (LARES et al., 2019) indicaron que el crecimiento económico de las familias cacaoteras en Piura, así como la sostenibilidad de su cadena, están relacionados principalmente con las estrategias de rescate y conservación de su material genético, llevadas a cabo por organizaciones de productores como APPROCAP. Esto es relevante debido a que es en los mercados nicho que resaltan las variedades nativas de cacaos donde se puede obtener mayor rentabilidad para un tipo de cacao de baja productividad y menores hectáreas como sucede en Piura. Además, porque son precisamente este tipo de cacaos los que se encuentran en riesgo de desaparecer, poniendo en riesgo la diversidad de este material genético, debido a la introducción de cacaos foráneos para incrementar la productividad.

Asimismo, la sostenibilidad de la cadena agroproductiva de cacao en Piura ha demostrado que las estrategias de conservación son más efectivas cuando han sido lideradas por organizaciones. Además, la diversificación productiva que realizan los socios productores de cooperativas como APPROCAP, para formar lotes con diferentes perfiles de sabor que se dirijan a mercados nicho, son acciones también fundamentales para asegurar la sostenibilidad de esta cadena.

Así, la sostenibilidad de la cadena agroproductiva de cacao en Piura se basa principalmente en la conservación de sus variedades nativas. Por eso, la importancia de su preservación y tomar de la antropología de la sostenibilidad, el facilitar las condiciones de cambio en las formas de producción, para favorecer la conservación de la biodiversidad y ponerla en valor.

\subsection{COOPERATIVA ACOPAGRO - REGIÓN SAN MARTÍN}

La Cooperativa Agraria Cacaotera Acopagro fue creada en el año 1997. Es una organización con 2,300 socios productores, con un rango de entre 945 a 3,000 kg de producción por hectárea y 5,000 toneladas de producción total de cacao por año. Su ámbito de acción se encuentra en las provincias de Mariscal Cáceres, Huallaga, Bellavista y Picota. Además, Acopagro es actualmente el primer productor de cacao orgánico del país. Desde el año 2008 trabajan bajo sistemas agroforestales y un programa de reforestación que ha

\footnotetext{
${ }^{8} \mathrm{http}: / /$ www.regionpiura.gob.pe/documentos/grde/revista_grde21.pdf
} 
favorecido la diversificación productiva para sus socios, en una visión también de conservación a largo plazo.

La información recogida durante las visitas de campo a esta cooperativa desde el año 2012 mostraron el estrecho vínculo que existe igualmente entre la asociatividad, la sostenibilidad de su cadena agroproductiva de cacao y su posicionamiento en mercados nichos internacionales, sobre todo los vinculados al Comercio Justo y los mercados orgánicos.

Desde la creación de la cooperativa, la cadena agroproductiva de cacao, relacionada con el impulso de la asociatividad en la región, permitió dar sostenibilidad económica y social a sus socios. La misma surgió en un contexto de terrorismo y narcotráfico desde la década de los años ochenta y que pudo ser controlado a través de estrategias de erradicación y programas de desarrollo alternativo. Los mismos se realizaron introduciendo cacaos foráneos, pero altamente productivos y con perfiles de sabor intenso.

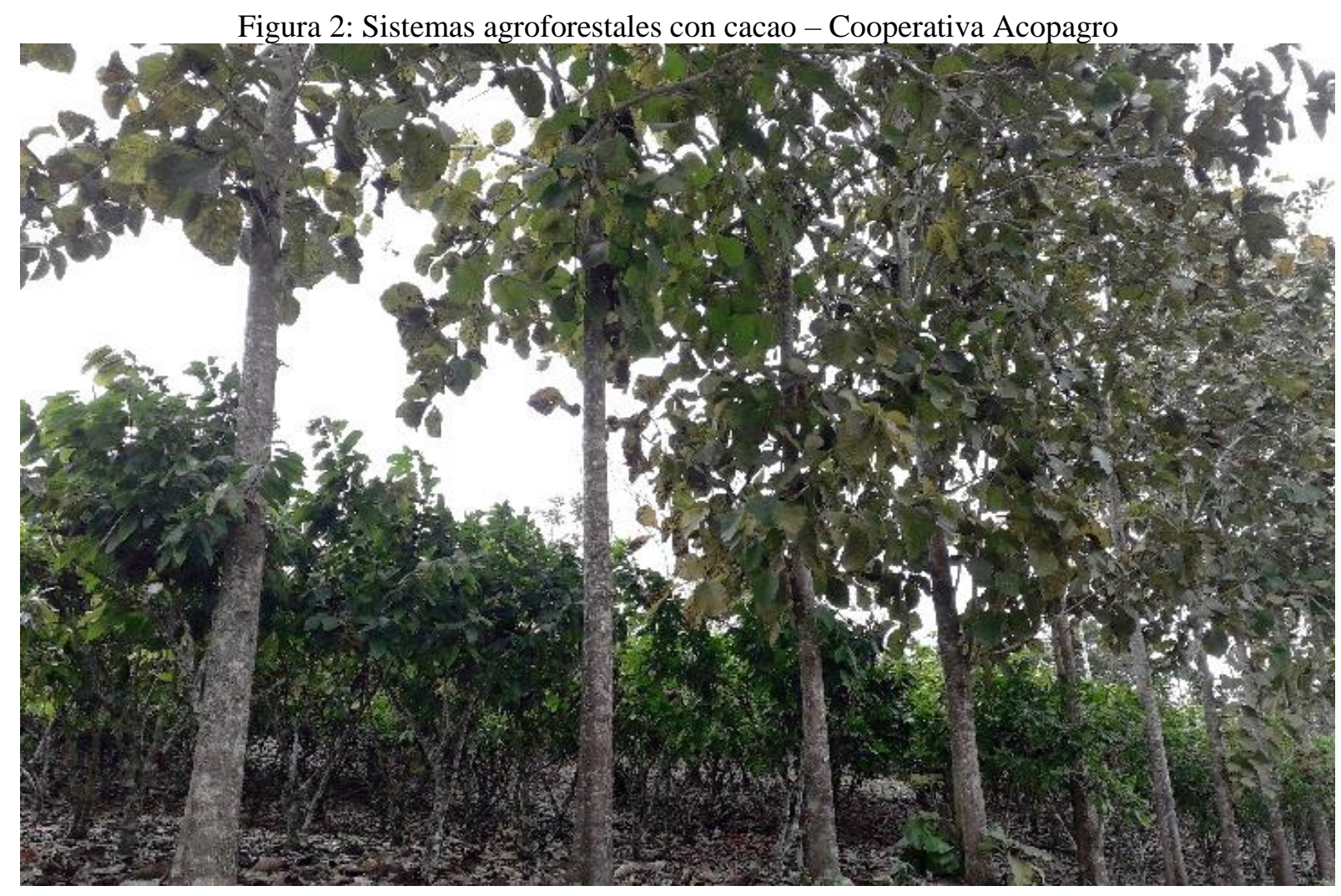

De igual manera, la sostenibilidad de la cadena se consolidó debido a la experiencia agraria de sus socios productores, población migrante casi en su totalidad, asentada en el ámbito de acción de la cooperativa, así como por su interés por encontrar una forma de generar ingresos lícitos, aunque menos rentables, en comparación con el cultivo de coca. A 
pesar de ello, obtuvieron beneficios económicos y sociales para los socios que les permitió un nivel de autonomía y especialización de sus capacidades productivas como en los procesos de postcosecha.

A pesar de que Acopagro trabaja con variedades de cacaos que no son nativos sí son altamente productivos en comparación con los de Piura. Su posicionamiento a nivel internacional en los mercados nicho antes mencionados, resaltan también sus características de calidad, como resultado del aprendizaje técnico a partir de la asistencia a los socios productores. Este factor diferencia, ha sido también un factor importante para la sostenibilidad de esta cadena agroproductiva a nivel regional.

Actualmente, la mayor predisposición de los socios productores a incorporar con mayor énfasis la dimensión ambiental en su cadena agroproductiva - reflejada en su participación en el programa de reforestación - se debe a que su lógica productiva se encuentra ya relacionada a las prácticas de producción acordes al cuidado del medio ambiente. Sin embargo, esta se basa también, y sobre todo, en las expectativas de rentabilidad a partir de los resultados ya obtenidos en su actividad productiva principal, la producción de cacao, en donde la dimensión económica sigue siendo la más importante.

En ese sentido, la sostenibilidad de la productividad del cultivo en ACOPAGRO, reflejada en mayores ingresos económicos para sus socios, ha sido un factor determinante en su decisión de participar en otras actividades productivas como el programa de reforestación.

\section{CONCLUSIONES}

Las semejanzas encontradas en la sostenibilidad de las cadenas agroproductivas de ambas cooperativas se basa en que han sabido aprovechar sus diferencias cualitativas y colocarse en mercados nicho que valoren esta diferenciación. En el caso de APPROCAP, el mercado de cacaos nativos resulta fundamental debido a su baja productividad. En el caso de ACOPAGRO, los mercados orgánicos y de calidad han sido los que mejor han favorecido su sostenibilidad.

Otro factor que ha favorecido la sostenibilidad de ambas cadenas agroproductivas, ha sido la respuesta positiva de los socios productores al desarrollo de capacidades en ambos contextos. Esto ha sido importante para dar continuidad a las características diferenciales de estos cacaos que resaltan su origen y calidad, así como para favorecer su productividad.

En ese sentido, como señala Vidal (2015), se ha agregado valor al producto para hacerlo sostenible, dándole competitividad no solo a él sino también a los productores. 
Los procesos de asociatividad en ambas regiones también han sido un elemento común que han favorecido la sostenibilidad de sus cadenas agroproductivas de cacao. La formación de estas organizaciones como cooperativas, con apoyo de la cooperación internacional en ambos casos, ha mostrado buenos resultados a nivel social y en rendimientos económicos. Esto porque la asociatividad ha garantizado la estandarización y calidad de los procesos productivos, así como su posibilidad de obtener mejores precios, con pagos extra por las certificaciones de Comercio Justo o Fair Trade.

Además, porque la asociatividad ha permitido darles un importante nivel de autonomía a ambas organizaciones, sobre todo a nivel comercial, que les ha permitido también posicionarse en diferentes mercados especiales a nivel internacional.

Todas estas características han permitido la sostenibilidad de la cadena agroproductiva de cacao en ambas regiones, la misma que se ha visto reflejada en el incremento de ingresos económicos para las familias de los socios productores. Este resultado, ha sido el principal motivo que ha favorecido la continuidad productiva en ambas cadenas agroproductivas, a pesar de los diferentes contextos y dificultades propias en cada zona.

Las políticas desde el Estado sobre cacao en el Perú deben tener en cuenta todas las regiones productoras, no solo la Amazonía. A pesar de que esta región posee la mayor producción nacional, en gran parte como consecuencia de los programas de desarrollo alternativo impulsados en los años noventa, no se debe dejar de considerar las variedades de cacaos nativos también presentes en las diferentes regiones del país. Esto porque uno de los factores importantes para la sostenibilidad de esta cadena es el gran potencial de comercialización y de generación de mejores ingresos económicos a partir de características organolépticas únicas que los cacaos nativos obtienen en mercados nicho.

\section{REFERENCIAS}

BANCO MUNDIAL. Gaining Momentum in Peruvian Agriculture: Opportunities to Increase Productivity and Enhance Competitiveness, 2017.

BRIGHTMAN, M.; LEWIS, J. The Anthropology of Sustainability: Beyond Development and Progress. En: The Anthropology of Sustainability. (pp 1-33). Palgrave Studies in Anthropology of Sustainability, 2017.

FORT, R.; VARGAS, R. Estrategias de articulación de los productores agrarios en la costa peruana. En: Agricultura peruana: nuevas miradas desde el Censo Agropecuario/Javier Escobal, Ricardo Fort y Eduardo Zegarra (Eds.). Lima: GRADE, 2015.

GROBER, U. Sustainability: A Cultural History. Totnes: Green Books, 2012. 
INSTITUTO NACIONAL DE ESTADÍSTICA E INFORMATICA (INEI) Perú Compendio Estadístico 2018 (Capítulo 13: Agrario) En:

https://www.inei.gob.pe/media/MenuRecursivo/publicaciones_digitales/Est/Lib1635/compendi o2018.html. 2018

LARES, L; CASTILLO, M. Desarrollo y sostenibilidad de la cadena agroproductiva del cacao piurano a partir de la conservación de sus variedades nativas: El caso de las cooperativas APPROCAP y Norandino. Consorcio de Investigación Económico y Social (CIES) / Centro de Investigación y Promoción del Campesinado (CIPCA) 2019.

Ministerio de Agricultura (MINAGRI). Plan Estratégico Cadena Productiva de Cacao. IICA, CICDA y GTZ. 2007

Ministerio de Agricultura (MINAGRI). Estrategia Nacional de Agricultura Familiar 2015 - 2021, 2015

PAJARES, E; LORET DE MOLA, C. Decolonizar el discurso del desarrollo sustentable. La narrativa de América Latina, 2016

PAREDES, H.; FORT, R. En los márgenes del boom agroexportador: articulación de los pequeños productores a las cadenas de valor globales. En: Perú: el problema agrario en debate. SEPIA XVII / Seminario Permanente de Investigación Agraria. Lima, SEPIA, 2018.

VIDAL, R. Cómo crear cadenas productivas competitivas y sostenibles: aprendizajes del biocomercio en Ecuador. En: DEBATES IESA. Volumen XX Número 2 Abril - Junio, 2015.

WILDLEY, A. De la coca al cacao: un análisis etnográfico sobre las nuevas tendencias del desarrollo alternativo en la Amazonía peruana. El caso de la Alianza Cacao Perú en el caserío Miguel Grau, Neshuya - Ucayali. Pontificia Universidad Católica del Perú (PUCP), 2016. 